Provided for non-commercial research and education use. Not for reproduction, distribution or commercial use.

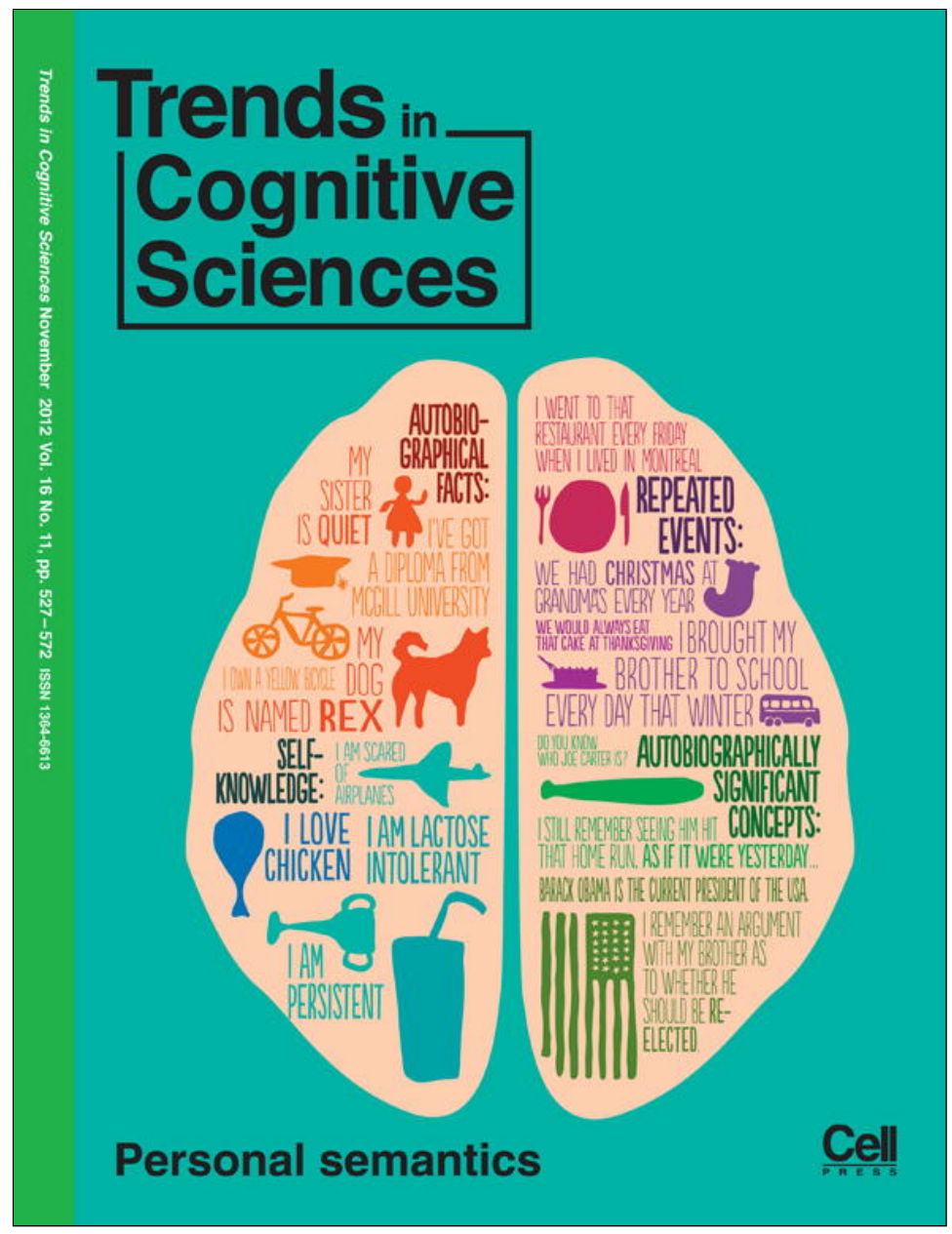

This article appeared in a journal published by Elsevier. The attached copy is furnished to the author for internal non-commercial research and education use, including for instruction at the authors institution and sharing with colleagues.

Other uses, including reproduction and distribution, or selling or licensing copies, or posting to personal, institutional or third party websites are prohibited.

In most cases authors are permitted to post their version of the article (e.g. in Word or Tex form) to their personal website or institutional repository. Authors requiring further information regarding Elsevier's archiving and manuscript policies are encouraged to visit:

http://www.elsevier.com/copyright 


\title{
Personal semantics: at the crossroads of semantic and episodic memory
}

\author{
Louis Renoult ${ }^{1,2}$, Patrick S.R. Davidson ${ }^{2}$, Daniela J. Palombo ${ }^{1,3}$, Morris Moscovitch ${ }^{1,3}$, \\ and Brian Levine ${ }^{1,3,4}$
}

${ }^{1}$ Rotman Research Institute, Baycrest, Toronto, Ontario M6A 2E1, Canada

${ }^{2}$ School of Psychology, University of Ottawa, Ontario K1N 6N5, Canada

${ }^{3}$ Department of Psychology, University of Toronto, Ontario M5S 3G3, Canada

${ }^{4}$ Department of Medicine, Division of Neurology, University of Toronto, Ontario M5S 1A1, Canada

\begin{abstract}
Declarative memory is usually described as consisting of two systems: semantic and episodic memory. Between these two poles, however, may lie a third entity: personal semantics (PS). PS concerns knowledge of one's past. Although typically assumed to be an aspect of semantic memory, it is essentially absent from existing models of knowledge. Furthermore, like episodic memory (EM), PS is idiosyncratically personal (i.e., not culturally-shared). We show that, depending on how it is operationalized, the neural correlates of PS can look more similar to semantic memory, more similar to EM, or dissimilar to both. We consider three different perspectives to better integrate PS into existing models of declarative memory and suggest experimental strategies for disentangling PS from semantic and episodic memory.
\end{abstract}

PS: an intermediate form of declarative memory?

Declarative memory is typically described as consisting of two systems, EM and semantic memory. EM entails recollecting unique events within their specific spatio-temporal context, imbued with a sense of the self mentally traveling through time to re-experience the original event [1]. By contrast, semantic memory pertains to culturally-shared general knowledge (including facts and vocabulary), detached from its context of acquisition and devoid of any subjective sense of mental time travel [1-3].

Although drawing a stark contrast between episodic and semantic memory has proved useful in spurring cognitive neuroscience research, these systems have largely been investigated in isolation, leading to an under-appreciation of their interactions and of potentially intermediate forms of memory. One such form of memory is personal semantics (PS). In early case studies, this type of memory was described in patients who could retrieve few or no EMs, yet showed knowledge of events from their personal past [4-7], resembling a 'skeleton autobiography' [5] that seemed 'to be drawn entirely from a personal pool of generalized knowledge' [4].

The paradox of PS is that it is highly personal (like EM), yet, at the same time, devoid of any subjective sense of recollection and detached from its context of acquisition (like semantic memory). Crucially, although PS is typically

Corresponding author: Levine, B. (blevine@research.baycrest.org). assumed to be a form of semantic memory, formal studies of PS have been rare and PS is not well integrated into existing models of semantic memory and knowledge (e.g., $[3,8,9])$. In the present review, we describe four ways in which PS has been operationalized in previous studies and show that how one defines PS can influence how it compares to EM and general semantic memory (GS). We then consider three different perspectives to better integrate PS into existing models of episodic and semantic memory, and offer suggestions for future work.

\section{When PS appears similar to semantic memory (and dissimilar to EM): autobiographical facts}

The most common conceptualization of PS is as a set of autobiographical facts (e.g., 'Nicholas is the name of my youngest brother.') in contrast to general knowledge of the world (e.g., 'Nicholas was a $4^{\text {th }}$-century saint.') and EMs of unique events ('I could see that my brother Nicholas' hands were shaking during his speech at my wedding'). Autobiographical facts share with EM the notion of personal content. However, in PS this personal content refers to a common organizing theme, whereas in EM it refers to a subjective first-person perspective [10]. In addition, autobiographical facts, like GS facts but unlike EMs, are detached from their original context of acquisition [11].

When PS is studied using autobiographical facts, it appears similar to GS and dissimilar to EM (Table 1). In priming studies with healthy people, both autobiographical and general facts can be primed by related knowledge and can be verified by participants more quickly than can EMs [12,13]. In neuropsychological studies using the Autobiographical Memory Interview (AMI; [14], Box 1), the typical finding is of impaired EM with relatively preserved PS and GS [7,15-18], especially in patients with medial temporal lobe (including hippocampal) lesions. A few studies have described the opposite pattern, that is, impaired PS and GS despite relatively preserved EM $[19,20]$, most often following extensive lesions or degeneration in lateral temporal regions.

Although the aforementioned neuropsychological studies suggest that PS and GS are usually impaired or preserved together, the few extant neuroimaging studies that have compared PS, GS, and EM have reported similar, but distinct neural correlates for PS and GS. In a series of 
Table 1. The four operational definitions of PS and their main characteristics

\begin{tabular}{|c|c|c|c|c|}
\hline & Autobiographical facts & Self-knowledge & Repeated events & AS concepts ${ }^{a}$ \\
\hline Examples & $\begin{array}{l}\text { My brother's } \\
\text { name is Nicholas }\end{array}$ & I am a stubborn person & $\begin{array}{l}\text { I brought my brother } \\
\text { to school every day }\end{array}$ & $\begin{array}{l}\text { Knowledge that Barack } \\
\text { Obama is President }+ \\
\text { Recollection of an } \\
\text { argument with my } \\
\text { brother as to whether } \\
\text { he should be re-elected }\end{array}$ \\
\hline $\begin{array}{l}\text { Typical neural } \\
\text { correlates }\end{array}$ & $\begin{array}{l}\text { MPFC, retrosplenial } \\
\text { cortex, temporal pole, } \\
\text { posterior temporal cortex }\end{array}$ & $\begin{array}{l}\text { MPFC, retrosplenial cortex, } \\
\text { precuneus, middle and inferior } \\
\text { temporal gyri, inferior } \\
\text { parietal lobe }\end{array}$ & $\begin{array}{l}\text { MPFC, hippocampus, } \\
\text { para-hippocampal gyrus, } \\
\text { temporo-parietal junction, } \\
\text { fusiform gyrus, inferior } \\
\text { temporal cortex }\end{array}$ & $\begin{array}{l}\text { MTL, temporo-parietal } \\
\text { junction, ventrolateral } \\
\text { prefrontal cortex, fusiform } \\
\text { gyrus }\end{array}$ \\
\hline $\begin{array}{l}\text { Neuropsychological } \\
\text { patterns }\end{array}$ & Similar to GS & Different from GS and EM & Similar to EM & Similar to EM \\
\hline
\end{tabular}

${ }^{a}$ Abbreviations: AS concepts, autobiographically significant concepts; GS, general semantic memory; EM, episodic memory; MPFC, medial prefrontal cortex; MTL, medial temporal lobe.

studies using sentence verification, Maguire and colleagues reported that, even though PS and GS-related activations overlapped in lateral temporal and medial prefrontal regions [Brodmann area (BA) 10], the PS condition showed greater activation relative to GS in a left-lateralized network including the medial prefrontal cortex, retrosplenial cortex, temporal pole, and temporoparietal junction [21,22]. A concordant structural MRI study in Alzheimer's patients reported a correlation between temporopolar volumes (especially on the left side) and PS as measured by the AMI [23]. Autobiographical facts are typically relatively idiosyncratic (e.g., I know that my brother owns an old blue station wagon), which is compatible with the presumed role of the temporal pole in the retrieval of information associated with unique objects and individuals

\section{Box 1. Two common methods for assessing PS in autobiographical memory: the Autobiographical Memory Interview and the Autobiographical Interview}

The Autobiographical Memory Interview (AMI) [14] refers to PS as 'factual knowledge about a person's own past', which corresponds best to the category of autobiographical facts in our classification scheme of operational definitions. In the AMI, PS is evaluated using questions about decontextualized facts, such as names of schools one attended, names of friends and colleagues, and addresses where one lived. One of the AMI's main goals is to assess PS and EM in a similar manner across the same three time periods: childhood, early adult life, and recent years. Although the AMI enables the comparison of PS and EM, the standard version does not contain a GS condition. For example, in the original study [14], PS was implicitly considered as part of GS, although GS was not formally assessed by means other than a famous personalities test. Subsequent neuropsychological studies using the AMI have shown that PS and GS (assessed by other means) are often preserved or impaired together in patients (see main text), suggesting that their neural bases are similar. However, the fact that GS is not examined formally in the AMI makes it more difficult to compare PS, EM, and GS with one another.

A different approach is taken in Levine et al.'s [72] Autobiographical Interview (Al) in which participants' descriptions of autobiographical events are scored for episodic and semantic details (among other types). No attempt is made to differentiate these types of memory from one another at the time of the interview; this is done only at the time of scoring. In the standard version, semantic details are not separated into PS versus GS categories (but see [38] for a modified version that evaluates PS as repeated events). However, the Al method could easily be modified to probe memory for PS details more specifically. (e.g., [24,25]). The greater medial prefrontal activation for PS than for GS in Maguire's neuroimaging studies is consistent with the putative role of this brain region in self-referential processing [26]. Interestingly, compared to EM, PS has been associated with lesser activity in these three brain regions (i.e., medial prefrontal cortex, temporal pole, and retrosplenial cortex), as well as in the hippocampus $[21,22,27]$. Taken together, these studies suggest that this form of PS has more in common with GS than with EM and are compatible with the idea of PS being a sub-domain of GS, with overlapping but partly distinct neural bases (Box 2). On the other hand, the fact that some brain regions (i.e., the medial prefrontal cortex, temporal pole and retrosplenial cortex) are differentially responsive to the three types of memory, with a decreasing pattern of activation from EM to PS to GS, is compatible with the idea of PS being an intermediate system on a continuum of abstraction from EM to GS (Box 3).

\section{When PS appears similar to EM (and dissimilar to GS): repeated events and autobiographically significant concepts}

Repeated events

A typical EM concerns a single personal experience, whereas semantic memory is based on the extraction of similarities across many events. PS is sometimes operationalized as intermediate between these two poles: repeated personal experiences (e.g., bringing my brother to school every day; Table 1). For such events, it is assumed that one typically does not remember a single instance, but instead has extracted the common characteristics from the series of similar but separate episodes ([28]; but see Box 4). Repeated events are generally remembered less vividly and associated with lower ratings of personal significance and emotion than are unique episodes [29-31]. On the other hand, memory for repeated events appears to be similar to memory for unique events in some ways: both usually contain visual imagery [32,33] and contextual information [34], often from a first-person perspective [35]. Accordingly, it has been proposed that EM may mediate recollection not only for unique events, but also for repeated or summarized events [36,37].

The few extant neuropsychological studies of memory for repeated events have observed parallel perturbations of 


\section{Box 2. PS as a sub-domain of semantic memory}

Is PS simply an aspect of GS, with similar organizing principles? This is a parsimonious assumption made by many investigators. On the face of it, the case in which this argument seems easiest to make is when PS is operationalized as autobiographical facts, but even here there are some differences between PS and GS in terms of their neural correlates. It may be that PS is a sub-domain of GS with overlapping, but partly distinct neural correlates. This is compatible with the picture of a complex, distributed semantic system in the brain, with different categories of objects (e.g., animals, tools, faces) represented preferentially by different brain regions $[9,73,74]$. PS may be one such category. A PS category in the semantic system would likely have played a useful role in evolution, a criterion that is considered essential for category specificity [74]. In functional neuroimaging studies, medial prefrontal, temporal polar, retrosplenial, and precuneus regions seem to be critical for the representation of personal aspects of semantic memory (Figure I). The medial prefrontal cortex, heavily involved in self-referential processing [26], is active in the most abstract forms of PS, namely autobiographical facts and self-knowledge (with activity centered around BA 10 in both cases). The temporal poles, known to support the representation of unique entities (e.g., [24,25]), are one of the brain regions most robustly associated with the representation of autobiographical facts. Finally, the retrosplenial cortex and precuneus, considered to play an important role in the interface of GS and EM [3], show greater activity for self-knowledge (as well as for autobiographical facts, in the case of retrosplenial cortex) than for GS. Importantly, these areas are coactive with other brain regions associated with GS, such as posterior and inferior temporal lobe and inferior parietal lobe, which is compatible with a conception of PS as a sub-domain of semantic memory. Information about personal relevance could thus be appended to existing semantic information [12]. Alternatively, for other types of PS, associated EMs could be added to semantic knowledge. This is most clearly seen in autobiographically significant concepts, which seem to be represented both in semantic and episodic memory $[44,45]$, and thus constitute an amalgamation between GS and EM rather than a sub-system of GS.
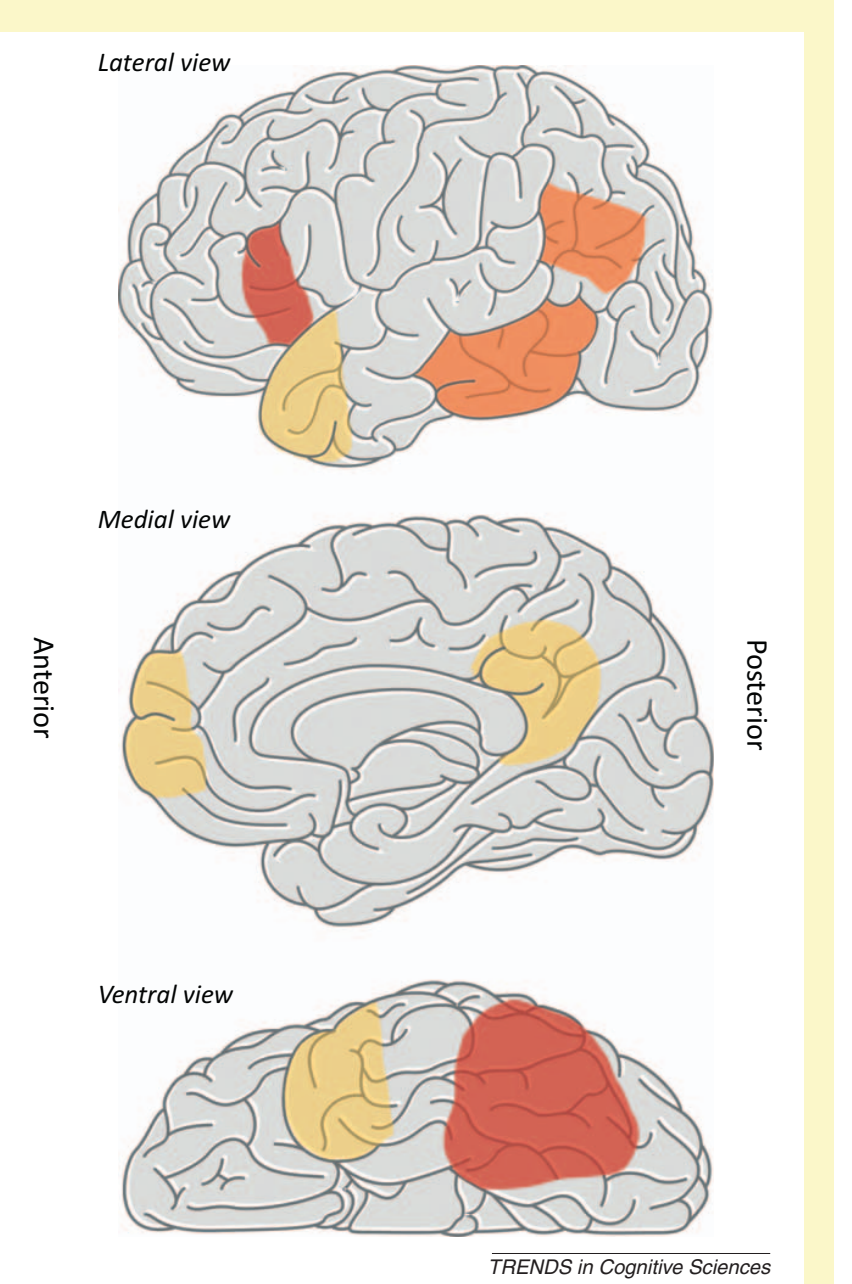

Figure I. Personal semantics (PS) as a sub-domain of semantic memory. Schematic view of the brain with lateral, medial, and ventral orientations. Areas in red show the approximate locations of the regions typically implicated in general semantic (GS) processing of concrete objects $([2,9]$; see also the semantic convergence zones in [3]). In yellow are the approximate locations of regions that show greater activity for the more abstract forms of PS (autobiographical facts and self-knowledge) than for GS. In orange are the approximate locations where GS and PS overlap.

from both GS and EM, this form of PS has much more in common with EM than with GS. Therefore, even though the dearth of studies on PS has sometimes resulted in our treating autobiographical facts and repeated events as belonging to the same category of semantic autobiographical memory [42], this may not be appropriate. Memory for unique and repeated events can be thought of as relying on different weightings of the same component processes (Box 5). Although they both include spatial and temporal features, as well as a subjective sense of re-experiencing events, unique events typically include more elaborated sensory imagery (perhaps owing to greater precuneus activation) and self-reflection (perhaps due to greater medial prefrontal cortex activation) than do repeated events.

\section{Autobiographically significant concepts}

A third way to study PS is through autobiographically significant concepts, that is, semantic concepts that are events. Taken together, these studies suggest that, although memory for repeated events can be differentiated 


\section{Box 3. The continuum perspective}

A number of continua have been proposed to describe how declarative memory, autobiographical memory, and knowledge are organized. In each of these domains, the continuum ranges from the specific to the general, in terms of the kind and/or quality of information represented: in declarative memory models, the range is from episodic to semantic memory [75-79]; in autobiographical memory models it is from specific episodes to summarized/repeated events to abstract knowledge ('life time periods'; [34]; see also [80]); in knowledge/semantic memory models [81-84], it is from more specific and context-bound semantic representations to more abstract and context-free ones. Perhaps PS should be thought of as an intermediate step between the episodic and the semantic memory systems (Figure I). In all of the aforementioned models, the most abstract information is also the least personally relevant. According to this perspective, the personal aspects of semantic memory may generally be less abstracted than GS (e.g., my collie vs the general category of collies) and have privileged interactions with specific episodes. Indeed, Conway ([85]) found that only less abstracted semantic categories could prime the retrieval of EMs. These results are complemented by recent neuroimaging studies that show that certain inherently autobiographical semantic categories ('names of friends') are associated with hippocampal/medial temporal activations [86,87], brain regions usually associated with episodic retrieval.

If one considers PS as an intermediate system in a continuum of abstraction from GS to EM, one might expect to see quantitative rather than qualitative variations in brain activity across these memory types. Indeed, several neuroimaging studies have found a graded decreasing pattern of activity from EM to autobiographical facts to GS in medial prefrontal, temporal polar and retrosplenial cortex $([21,22,27]$, see also [42]). The precuneus also shows a graded reduction of activity from EM to self-knowledge to GS [63]. However, the interpretation of these observations is complicated by the fact that very few studies have included all three types of memory (i.e., PS, GS and EM). A further problem with the continuum perspective is that there may still be a qualitative gap between the less abstracted forms of personal knowledge (i.e., repeated events) and EM, notably in term of time frame (shorter for episodes), related awareness (noetic vs autonoetic awareness, Box 4), and visual imagery (generic for PS vs detailed for EM).

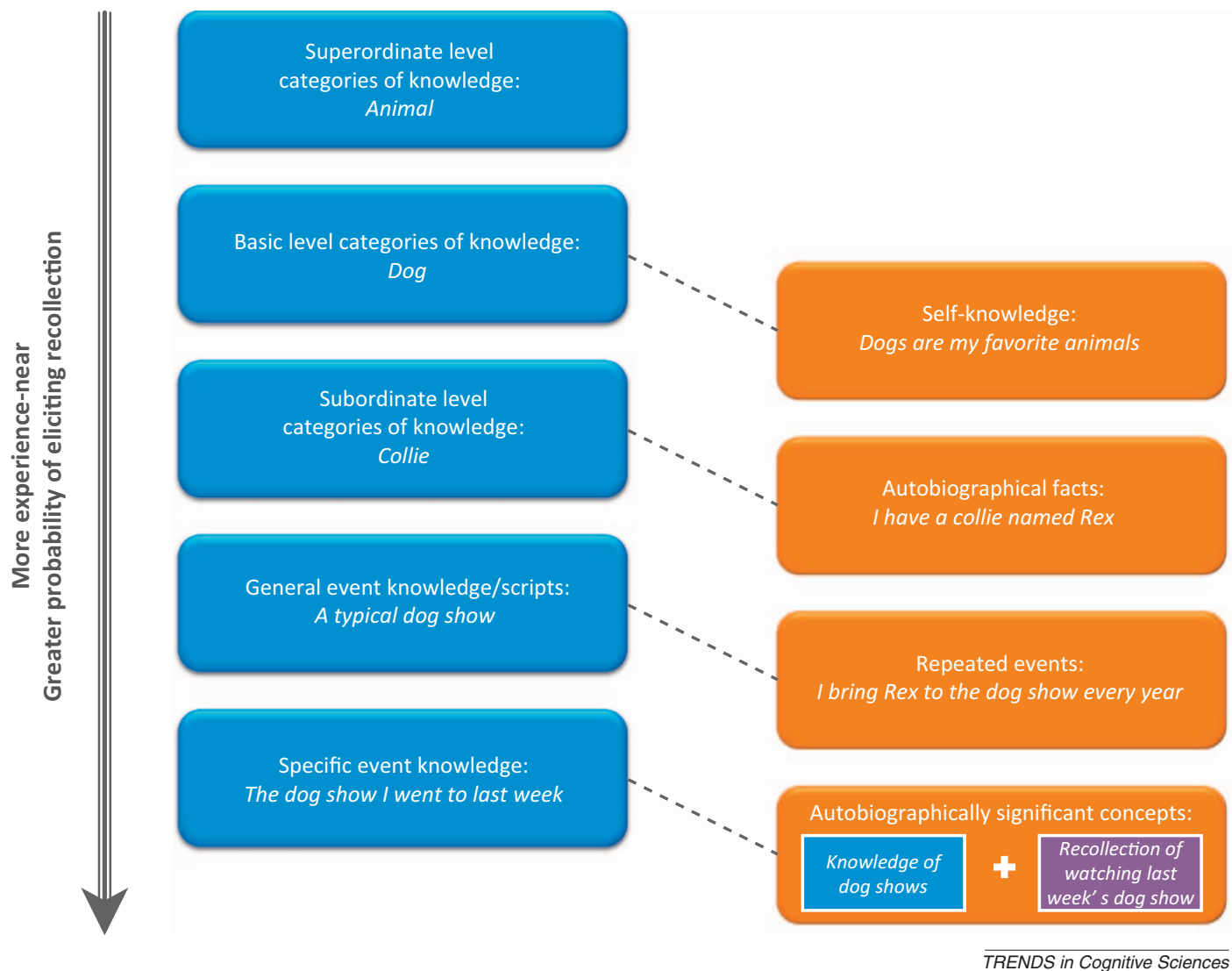

Figure I. The continuum perspective. This figure represents a hypothetical continuum of content in general semantics (GS, in blue) and personal semantics (PS, in orange). In both GS and PS, representations become less and less abstracted and more and more context-dependent, from the higher to the lower levels of representation (see [81]). According to this perspective, the personal aspects of semantic memory may be less abstracted than GS (but still related in content, as indicated by the dotted lines). Moreover, in GS itself, less abstracted representations are also more experience-near and thus more personal, while still being part of semantic knowledge (i.e., knowledge of events such as 'The dog show I went to last week.' [34]). Autobiographically significant concepts are semantic concepts that are associated with vivid episodic memories (EMs). In this example, the concept of 'dog shows' becomes autobiographically significant by virtue of its association with episodic recollection of last week's dog show (in purple). Episodic recollection can be associated with concepts at a less abstracted level of representation, although this becomes less likely as abstraction increases.

associated with vivid EMs from our lives. Conceptually, this form of PS is at the intersection of GS and EM (Table 1). In contrast to other forms of PS, it does not relate to knowledge of personal events or the self, but rather to semantic information, which, by its tight association with specific episodes, has high degrees of personal significance and emotional salience [43].

In initial work on this phenomenon with healthy people, Westmacott and Moscovitch [44] showed that famous names that brought to mind personally-significant EMs 


\section{Box 4. Participants in psychology experiments do not necessarily access the representations we assume they do}

It is always difficult to be certain that one is making the correct inferences about underlying cognitive processes based on behavior. For instance, single episodes can later be retrieved as autobiographical facts: I may know what occurred during my brother's speech at my wedding, without having a perceptually rich, first-person reexperiencing of it. In other words, this single event could be retrieved with noetic awareness, that is, the type of awareness that is associated with knowing about the world and retrieving semantic knowledge, rather than with autonoetic awareness, that is, the type of awareness associated with subjectively re-experiencing events from EM [1]. As Tulving cautioned, there is always the 'possibility of [our] answering questions directed to episodic memory on the basis of our general knowledge of the world' ([91]; see also[32]). According to this view, even if the probability of autonoetically re-experiencing events in their original episodic context is greater for unique instances, both single and repeated events can be associated with either autonoetic or noetic remembering. To overcome this difficulty, the remember/know procedure [92], in which participants

were associated with superior performance on tests of semantic and episodic memory, compared to less personally-significant (but equally familiar) famous names. Westmacott et al. [45] (see also [43]) found that this performance advantage was absent in Alzheimer's and amnesic patients, two groups with severe EM impairment due to medial temporal lobe damage. By contrast, the advantage was preserved in semantic dementia patients, who have marked deficits in the ability to remember semantic concepts, stemming from lateral temporal lobe deterioration (see also [46] for similar results using public events instead of famous names). These results suggest that, although famous names are thought to be represented in semantic report on the type of awareness that they have experienced during retrieval, can be used, as can other methods (e.g., [93]). Alternatively, it would be interesting to train participants to retrieve memories voluntarily in either the noetic or the autonoetic mode. A prior selection of vivid memories of personal experiences would be necessary. The recall phase would then entail focusing either on the meaning and knowledge associated with a personal experience (third-person remembering) or on attempting to re-experience it in its original context (first-person recollection). In addition to allowing for a better control of retrieval mode, it would also allow for comparisons between very similar memories (e.g, unique events retrieved in the noetic vs autonoetic mode) matched in content and age between the two conditions (for a similar approach with the cueword technique see [94]). This enterprise, however, may face other challenges. Among them is the possibility that EM processes and information may be activated initially relatively automatically $[44,86,87]$, with the participant's awareness of this retrieved information emerging only at a later stage of processing.

memory, the performance advantage seen for autobiographically significant names may derive from the additional influence of EM.

A number of neuroimaging studies have investigated the impact of self-relevance on neural activity, comparing, for example, personally known versus unknown (or famous) faces, names, objects, or scenes (reviewed in [42]). However, in these studies, personally relevant stimuli typically differ from control stimuli not only by their association with EMs, but also by a greater degree of familiarity and associated knowledge. This confound was taken into account in an fMRI study by Denkova, Botzung and Manning [47], in which names and faces of famous

\section{Box 5. The component processes view}

The effective encoding and retrieval of declarative memories depends on multiple mnemonic and other processes, including attention, selfreflection, emotion, spatial and temporal processing, sensory-perceptual imagery, and executive functions $[78,88,89]$. One way to conceptualize how PS, GS, and EM fit together is to assume that these three types of memory simply rely on different weightings of the aforementioned component processes [90].

According to this view, EM might involve a greater degree of selfreflection, self-projection in time and space, emotion, and sensoryperceptual imagery than PS and GS (Table I). In turn, PS might differ from GS in terms of the former's greater involvement of selfreflection, and possibly a greater degree of emotional involvement. The precise differences between PS, EM, and GS will depend on how PS is operationalized. For example, some forms of PS, such as memory for repeated events, appear to contain spatial and temporal features, and may share with EM a chronological re-experiencing of the event from a first-person perspective. However, they might still differ from EM in terms of sensory imagery and self-reflection. Neuroimaging studies have indeed found greater activity for unique than repeated events in the precuneus and medial prefrontal cortex $[30,31,39]$, involved respectively in visuo-spatial processing and selfreflection. These forms of PS (e.g., bringing my brother to school every day) would be interesting to compare to elaborated scripts (e.g., going to school), which are considered part of GS [81]. It is likely that they would differ mainly in terms of a greater reliance on personal reflection for PS than for GS and possibly also in terms of more elaborated sensory imagery (instead of generic images). Finally, selfknowledge can be considered to involve a more abstract form of selfreflection than EM, with less sensory imagery and less rich subjective projection in time and space. Self-knowledge is the only form of PS which shows similar activation in medial prefrontal cortex to EM. In contrast, EM is associated with greater activity than self-knowledge in brain areas related to visuospatial processing, including the precuneus and superior and inferior parietal lobules.

Table I. The component processes view. The component processes view conceptualizes the different types of PS, GS, and EM as representing different weighting of various cognitive processes. The ' + ' sign represents the weighting of the relevant cognitive processes (more pluses correspond to greater weight).

\begin{tabular}{|c|c|c|c|c|c|c|}
\hline & GS & Autobiographical facts & Self-knowledge & Repeated events & AS concepts & EM \\
\hline Self-reflection & - & + & + & ++ & ++ & +++ \\
\hline Sensory-perceptual imagery & Poor & Poor & Poor & Generic & Detailed & Detailed \\
\hline Spatial/temporal features & + & + & + & ++ & ++ & +++ \\
\hline Emotional valence & - & + & ++ & + & ++ & +++ \\
\hline Possibility of eliciting recollection & Poor & $\begin{array}{l}\text { Poor } \\
\text { (may vary with recency) }\end{array}$ & Poor & Good & Good & Systematic \\
\hline Typical perspective & $3^{\text {rd }}$ Person & $3^{\text {rd }}$ Person & $3^{\text {rd }}$ Person & $1^{\text {st }}$ Person & $1^{\text {st }} / 3^{\text {rd }}$ Person & $1^{\text {st }}$ Person \\
\hline
\end{tabular}


people high or low in autobiographical significance - but equal in familiarity - were compared. The results of this study converged with the neuropsychological data to suggest that autobiographically significant concepts do indeed depend on the medial temporal lobe (see also [48]), although they also identified other brain regions, such as the left ventrolateral prefrontal cortex, the temporoparietal junction, the fusiform gyrus, and the lingual gyrus. These results were interpreted as indicating that autobiographically relevant concepts may involve more effortful retrieval (left prefrontal activation), the activation of richer autobiographical information (temporo-parietal junction), and the retrieval of sensory perceptual details (lingual gyrus) [47]. A recent event-related potential (ERP) study (Renoult et al., unpublished data) provides further evidence of the episodic nature of autobiographically significant concepts: compared to famous names for which participants had only general knowledge, autobiographically significant names were associated with increased amplitude of the late positive component (LPC), an ERP index of episodic recollection [49]. In contrast, the N400 component, an ERP index of semantic processing [50], did not differentiate the two kinds of names, but instead was related to the amount of semantic knowledge participants had of each famous person. These results suggest that autobiographically significant concepts differ from other concepts by being represented in EM, in addition to semantic memory. Crucially, as in the original paradigm [44,45], autobiographical significance was incidental to task performance as it was determined by a separate sample of participants or by the subjects themselves but at the end of the experiment. This suggests that the activation of relevant episodic information by these concepts is automatic and, more generally, that this information is an intrinsic part of autobiographically significant concepts.

Although not as well-studied as the other forms of PS, autobiographically significant concepts constitute a promising way to study interactions between GS and EM. The data available so far suggest that this form of PS, like memory for repeated events, has neural correlates that are similar to those of EM.

\section{When PS appears dissimilar to both GS and EM: self-knowledge}

A fourth tradition of work on PS focuses on the self, selfimage, and personal identity. For example, Neisser [51] proposed that the conceptual self is the most basic form of self-knowledge, allowing us to reflect on our personal characteristics and attributes (e.g., 'I am a fast reader, but poor at remembering names'). Similarly, Brewer [32] refers to the self-schema as a 'cognitive structure that contains generic knowledge about the self'. Self-knowledge has most often been operationalized as the summary of one's personality traits (e.g., 'I am a stubborn person' $[52,53])$. Because they focus on self-image, these aspects of PS may be strongly influenced by emotional and social factors both during encoding and retrieval [54], and could play a crucial role in maintaining a concept of self that would fit with the rememberer's goals and emotions [55]. In addition, self-knowledge may be differentiated from other forms of autobiographical knowledge by slower updating mechanisms [56]. These psychological characteristics, together with the neuropsychological data (see below), argue against the idea of self-knowledge being a subset of the category of autobiographical facts (Table 1).

Behavioral studies by Klein and collaborators have dissociated self-knowledge from EM and GS. In these studies, self-knowledge was operationalized as knowledge of one's personality traits. Using a priming design, Klein and Loftus [57] showed that judging the self-relevance of a trait (PS condition) did not influence the time required for participants to recollect an event in which they displayed that personality trait (EM condition). Similarly, judging whether a trait was self-relevant did not influence the time it took to define that trait (GS condition). These behavioral results, therefore, suggest a relative independence of trait self-knowledge from EM or GS related to these traits ([52]).

In neuropsychological work, Klein and colleagues showed that self-knowledge can be preserved when EM and GS are impaired ([58-60], see also [36]) and preserved even when knowledge of autobiographical facts is impaired $[60,61]$. This form of PS was considered to be intact when reasonable correlations were found between patients' selfreports about their personality traits and reports from family members. Klein and colleagues interpreted their results as indicating that self-knowledge may be a specialized subsystem within semantic memory.

Neuroimaging studies have found that self-knowledge, as operationalized by the self-evaluation of one's personality traits or by self-evaluative judgments ('I enjoy going to New Year's parties.'; [62-64]), is associated with neural correlates that are somewhat distinct from those of EM and GS. The medial prefrontal cortex (BA 10, extending into BA 9), posterior cingulate cortex (including the retrosplenial cortex), and precuneus have consistently shown greater activity for self-knowledge than for GS (operationalized as judging the valence of the traits [65-69] or as verifying statements of factual knowledge [62-64,70]). The same regions of the medial prefrontal cortex and posterior cingulate cortex were also found to be activated for EM (operationalized as verifying sentences about unique events [63] or as recognizing previously studied pictures [71]). In contrast, these same studies reported that EM was associated with greater activity than PS in brain regions related to visuo-spatial processing, such as the precuneus and the superior and inferior parietal lobules. Finally, selfknowledge was also found to activate brain regions often associated with GS [3], such as the left middle and inferior temporal gyrus and left inferior parietal lobe [64-66,71], and the left temporal pole [62]. Together, the neuropsychological and neuroimaging evidence suggests that selfknowledge is the form of PS that is the most easily dissociable from GS and EM, although further evidence is required. For example, the case for PS being truly independent of EM and GS would be stronger if the opposite neuropsychological dissociation from Klein and colleagues' [52] had ever been reported (that is, a patient with impaired self-knowledge, but intact EM and GS). In addition, the evidence of overlapping neuroimaging patterns for selfknowledge and GS is compatible with Klein et al.'s hypothesis that the self-knowledge aspect of PS is supported by semantic memory [52] (Box 2). 


\section{Concluding remarks}

Even though the empirical assessment of PS first began 30 years ago [4], this field is still in its infancy. Up to the present, the study of PS has largely been restricted to the autobiographical memory literature, as PS is typically not included in models of semantic memory or knowledge. The dearth of research on PS may have been due to the assumption made by many researchers that PS is highly similar to, or even synonymous with, GS. Here, we have shown that this is an oversimplification. Whereas some forms of PS (e.g., autobiographical facts) appear to have neural correlates that are very similar to GS, others (e.g., memory for repeated events and autobiographically significant concepts) have neural correlates that are much more similar to those of EM. Finally, self-knowledge is a

\section{Box 6. Current problems and questions for future research}

- Is there a qualitative gap between different forms of PS? To study PS, researchers have focussed separately on two factors: personal implication (i.e., comparison between personal and non-personal facts) and uniqueness (i.e., comparison of unique vs repeated events). Although, to our knowledge, autobiographical facts and repeated events have never been compared directly, there appears to be a qualitative gap between them in terms of their psychological and neural correlates, perhaps due to the inherent difficulty of contrasting facts and events. To situate PS better, it may be useful to study personal implication and uniqueness in the same experiment to allow for a comparison between the following categories: personal-unique, personal-repeated, nonpersonal-unique and non-personal-repeated events. Non-personal unique events could, for instance, be operationalized as the retrieval of movies or news from the same time period as the personal events. Non-personal repeated events could consist of the retrieval of scripts or of general knowledge associated with extended events.

- How do the more abstract forms of PS differ from GS? One interpretation of how autobiographical facts and self-knowledge differ from GS stems from the notion of expertise [57,95]. All individuals are experts on themselves, in that these types of knowledge are overlearned and accessed frequently. This could explain the resilience of self-knowledge to neural damage [52]. Indeed, neuropsychological data show that pre-morbid expertise in certain knowledge categories can lead to a selective preservation of these categories in patients with semantic impairments $[96,97]$. Accordingly, differences in brain activation between PS and GS could be influenced by variations in category expertise. It would thus be interesting, when comparing self-knowledge and GS, to include GS categories in which participants are experts.

- Does PS only concern knowledge of facts and events from our personal past? Recent work suggests that people also possess general knowledge about facts and events that they anticipate to happen in their personal future $([98,99]$, e.g., 'We plan to record an album with my friend Phillippe and I imagine that I will spend a lot of time with him, composing new songs and rehearsing them in our room.'). It will thus be of interest to determine whether these future and past-oriented forms of PS have similar neural correlates.

- Is my table the same as your table? Semantic memory is generally considered to be culturally shared [1,2]. Even though clear individual differences in how people categorize the world have been described (e.g., [100-102]), the general topic of individual differences in semantic memory has largely been neglected. In neuroimaging studies, anatomical variations in the organization of knowledge have been reported and may explain inconsistent findings in studies of category specificity $[103,104]$. Thus, bridging the gap between the personal and non-personal forms of semantics may also require investigating inter-individual variation in processing general concepts. form of PS that seems to be at least partly dissociable from GS and EM.

Three broad conceptualizations of PS can be articulated: the GS, continuum, and component processes views, outlined in Boxes 2, 3 and 5. Each of these might best describe one or two of the four different operational definitions of PS we have outlined, but not others. For instance, knowledge of autobiographical facts, and perhaps self-knowledge, might best be viewed as subdomains/categories of semantic memory (Box 2). The more episodic types of PS, such as memory for repeated events, could perhaps most easily be integrated into models of declarative memory by proposing either a continuum of abstraction in knowledge representations (Box 3) or a component processes approach (Box 5). Although a continuum model would be supported by quantitative variations in brain activity seen in a number of regions when comparing EM, PS and GS [21,22,27,63], a component process view may better account for the qualitative gap that appears to exist between the less abstracted form of PS (such as memory for repeated events) and EM. At present, the data are insufficient to adjudicate among these different models of PS. Future work should distinguish among the four types of PS we have outlined here, because they seem to have at least somewhat different cognitive and neural bases from one another. Careful comparisons of these four forms of PS with one another and with EM and GS should help to shed light not only on the nature of PS but also on declarative memory and knowledge more broadly (Box 6).

\section{Acknowledgments}

Preparation of this manuscript was supported by the Heart and Stroke Foundation of Canada Centre for Stroke Recovery, fellowship 23710 from the 'Fonds de la Recherche en Santé du Québec' (FRSQ) to L.R., grants A8347 (M.M.) and 341649 (P.S.R.D.) from the Natural Sciences and Engineering Research Council of Canada (NSERC), and grant MOP62963 (B.L.) from the Canadian Institutes of Health Research (CIHR). We thank Patrick Seymour for his assistance with preparing the figures.

\section{References}

1 Tulving, E. (2002) Episodic memory: from mind to brain. Annu. Rev. Psychol. 53, 1-25

2 Martin, A. (2001) Functional neuroimaging of semantic memory. In Handbook of Functional NeuroImaging of Cognition (Cabeza, R. and Kingstone, A., eds), pp. 153-186, MIT Press

3 Binder, J.R. and Desai, R.H. (2011) The neurobiology of semantic memory. Trends Cogn. Sci. 15, 527-536

4 Cermak, L.S. and O'Connor, M. (1983) The anterograde and retrograde retrieval ability of a patient with amnesia due to encephalitis. Neuropsychologia 21, 213-234

5 Warrington, E.K. and McCarthy, R.A. (1988) The fractionation of retrograde amnesia. Brain Cogn. 7, 184-200

6 Tulving, E. et al. (1988) Priming of semantic autobiographical knowledge: a case study of retrograde amnesia. Brain Cogn. 8, 3-20

7 Levine, B. et al. (1998) Episodic memory and the self in a case of isolated retrograde amnesia. Brain 121, 1951-1973

8 Murphy, G.L. (2002) The Big Book of Concepts, MIT Press

9 Martin, A. (2007) The representation of object concepts in the brain. Annu. Rev. Psychol. 58, 25-45

10 Tulving, E. (1989) Remembering and knowing the past. Am. Sci. 77, 361-367

11 Larsen, S.F. (1992) Personal context in autobiographical and narrative memories. In Theoretical Perspectives on Autobiographical Memory (Conway, M.A. et al., eds), pp. 53-71, Kluwer Academic Publishers

12 Conway, M.A. (1987) Verifying autobiographical facts. Cognition 26, 39-58 
13 Conway, M.A. and Bekerian, D.A. (1987) Organization in autobiographical memory. Mem. Cogn. 15, 119-132

14 Kopelman, M.D. et al. (1989) The autobiographical memory interview: a new assessment of autobiographical and personal semantic memory in amnesic patients. J. Clin. Exp. Neuropsychol. 11, 724-744

15 Oxbury, S. et al. (1997) Severe amnesia: an usual late complication after temporal lobectomy. Neuropsychologia 35, 975-988

16 Viskontas, I.V. et al. (2000) Remote episodic memory deficits in patients with unilateral temporal lobe epilepsy and excisions. $J$. Neurosci. 20, 5853-5857

17 Hirano, M. et al. (2002) I cannot remember, but I know my past events: remembering and knowing in a patient with amnesic syndrome. $J$. Clin. Exp. Neuropsychol. 24, 548-555

18 McCarthy, R.A. et al. (2005) Remembering and forgetting of semantic knowledge in amnesia: a 16-year follow-up investigation of RFR. Neuropsychologia 43, 356-372

19 Hodges, J.R. et al. (1992) Semantic dementia. Progressive fluent aphasia with temporal lobe atrophy. Brain 115 (Pt 6), 1783-1806

20 Eslinger, P.J. (1998) Autobiographical memory after temporal lobe lesions. Neurocase 4, 481-495

21 Maguire, E.A. and Frith, C.D. (2003) Aging affects the engagement of the hippocampus during autobiographical memory retrieval. Brain 126, 1511-1523

22 Maguire, E.A. and Mummery, C.J. (1999) Differential modulation of a common memory retrieval network revealed by positron emission tomography. Hippocampus 9, 54-61

23 Gilboa, A. et al. (2005) Retrieval of autobiographical memory in Alzheimer's disease: relation to volumes of medial temporal lobe and other structures. Hippocampus 15, 535-550

24 Tranel, D. (2009) The left temporal pole is important for retrieving words for unique concrete entities. Aphasiology 23, 867

25 Ross, L.A. and Olson, I.R. (2011) What's unique about unique entities? An fMRI investigation of the semantics of famous faces and landmarks. Cereb. Cortex 22, 2005-2015

26 D'Argembeau, A. and Salmon, E. (2012) The neural basis of semantic and episodic forms of self-knowledge: insights from functional neuroimaging. Adv. Exp. Med. Biol. 739, 276-290

27 Maguire, E.A. et al. (2001) The effects of bilateral hippocampal damage on fMRI regional activations and interactions during memory retrieval. Brain 124, 1156-1170

28 Neisser, U. (1981) John Dean's memory: a case study. Cognition 9, 1-22

29 Addis, D.R. et al. (2004) Recollective qualities modulate hippocampal activation during autobiographical memory retrieval. Hippocampus $14,752-762$

30 Levine, B. et al. (2004) The functional neuroanatomy of episodic and semantic autobiographical remembering: a prospective functional MRI study. J. Cogn. Neurosci. 16, 1633-1646

31 Holland, A.C. et al. (2011) The neural correlates of specific versus general autobiographical memory construction and elaboration. Neuropsychologia 49, 3164-3177

32 Brewer, W.F. (1986) What is autobiographical memory? In Autobiographical Memory (Rubin, D.C., ed.), pp. 25-49, Cambridge University Press

33 Rubin, D.C. et al. (2003) Belief and recollection of autobiographical memories. Mem. Cogn. 31, 887-901

34 Conway, M.A. and Pleydell-Pearce, C.W. (2000) The construction of autobiographical memories in the self-memory system. Psychol. Rev. 107, 261-288

35 Robinson, J.A. and Swanson, K.L. (1993) Field and observer modes of remembering. Memory 1, 169-184

36 Tulving, E. (1993) Self-knowledge of an amnesic individual is represented abstractly. In The Mental Representation of Trait and Autobiographical Knowledge About the Self (Srull, T.K. and Wyer, R.S., eds), pp. 147-156, Erlbaum

37 Brewer, W.F. (1996) What is recollective memory? In Remembering our Past: Studies in Autobiographical Memory (Rubin, D.C., ed.), pp. 19-66, Cambridge University Press

38 St-Laurent, M. et al. (2009) Determinants of autobiographical memory in patients with unilateral temporal lobe epilepsy or excisions. Neuropsychologia 47, 2211-2221

39 Addis, D.R. et al. (2004) Characterizing spatial and temporal features of autobiographical memory retrieval networks: a partial least squares approach. Neuroimage 23, 1460-1471
40 Nadel, L. et al. (2007) Autobiographical memory retrieval and hippocampal activation as a function of repetition and the passage of time. Neural Plast. 2007, 90472

41 Svoboda, E. and Levine, B. (2009) The effects of rehearsal on the functional neuroanatomy of episodic autobiographical and semantic remembering: a functional magnetic resonance imaging study. J. Neurosci. 29, 3073-3082

42 Martinelli, P. et al. (2012) Neural substrates of the self-memory system: new insights from a meta-analysis. Hum. Brain Mapp. http://dx.doi.org/10.1002/hbm.22008

43 Westmacott, R. et al. (2001) Different patterns of autobiographical memory loss in semantic dementia and medial temporal lobe amnesia: a challenge to consolidation theory. Neurocase 7, 37-55

44 Westmacott, R. and Moscovitch, M. (2003) The contribution of autobiographical significance to semantic memory. Mem. Cogn. 31, 761-774

45 Westmacott, R. et al. (2004) The contribution of autobiographical significance to semantic memory: evidence from Alzheimer's disease, semantic dementia, and amnesia. Neuropsychologia 42, 25-48

46 Petrican, R. et al. (2010) Recollection and familiarity for public events in neurologically intact older adults and two brain-damaged patients. Neuropsychologia 48, 945-960

47 Denkova, E. et al. (2006) Neural correlates of remembering/knowing famous people: an event-related fMRI study. Neuropsychologia 44, 2783-2791

48 Viskontas, I.V. et al. (2009) Human medial temporal lobe neurons respond preferentially to personally relevant images. Proc. Natl. Acad. Sci. U.S.A. 106, 21329-21334

49 Rugg, M.D. and Curran, T. (2007) Event-related potentials and recognition memory. Trends Cogn. Sci. 11, 251-257

50 Kutas, M. et al. (2006) Psycholinguistics electrified II (1994-2005), In Handbook of Psycholinguistics (2nd edn) (Matthew, J.T. and Morton, A.G., eds), pp. 659-724, Academic Press

51 Neisser, U. (1988) Five kinds of self-knowledge. Philos. Psychol. 1, 35-59

52 Klein, S.B. and Lax, M.L. (2010) The unanticipated resilience of trait self-knowledge in the face of neural damage. Memory 18, 918-948

53 Craik, F.I.M. et al. (1999) In search of the self: a positron emission tomography study. Psychol. Sci. 10, 26-34

54 Northoff, G. et al. (2006) Self-referential processing in our brain - A meta-analysis of imaging studies on the self. Neuroimage 31, 440-457

55 Baddeley, A. (1992) What is autobiographical memory? In Theoretical Perspectives on Autobiographical Memory (Conway, M.A. et al., eds), pp. 13-29, Kluwer Academic Publishers

56 Wagenaar, W.A. (1992) Remembering my worst sins: How autobiographical memory serves the updating of the conceptual self In Theoretical Perspectives on Autobiographical Memory (Conway, M.A. et al., eds), pp. 263-274, Kluwer Academic Publishers

57 Klein, S.B. and Loftus, J. (1993) The mental representation of trait and autobiographical knowledge about the self. In Advances in Social Cognition (Vol. 5) Srull, T.K. and Wyer, R.S.,eds In pp. 1-49, Lawrence Erlbaum Associates

58 Klein, S.B. et al. (1999) Independence of episodic and semantic selfknowledge: the case from autism. Soc. Cogn. 17, 413-436

59 Klein, S.B. et al. (2002) Is there something special about the self? A neuropsychological case study. J. Res. Pers. 36, 490-506

60 Klein, S.B. et al. (2003) Preserved knowledge of self in a case of Alzheimer's dementia. Soc. Cogn. 21, 157-165

61 Klein, S.B. et al. (2002) Memory and temporal experience: the effects of episodic memory loss on an amnesic patient's ability to remember the past and imagine the future. Soc. Cogn. 20, 353-379

62 Schroeter, M.L. et al. (2010) Traumatic brain injury affects the frontomedian cortex - an event-related fMRI study on evaluative judgments. Neuropsychologia 48, 185-193

63 Zysset, S. et al. (2002) The anterior frontomedian cortex and evaluative judgment: an fMRI study. Neuroimage 15, 983-991

64 Zysset, S. et al. (2003) Functional specialization within the anterior medial prefrontal cortex: a functional magnetic resonance imaging study with human subjects. Neurosci. Lett. 335, 183-186

65 D'Argembeau, A. et al. (2010) The neural basis of personal goal processing when envisioning future events. J. Cogn. Neurosci. 22, 1701-1713 
66 D'Argembeau, A. et al. (2010) Modulation of medial prefrontal and inferior parietal cortices when thinking about past, present, and future selves. Soc. Neurosci. 5, 187-200

67 Schmitz, T.W. et al. (2004) Metacognitive evaluation, self-relevance, and the right prefrontal cortex. Neuroimage 22, 941-947

68 Schmitz, T.W. et al. (2006) Neural correlates of self-evaluative accuracy after traumatic brain injury. Neuropsychologia 44, 762-773

69 Whitfield-Gabrieli, S. et al. (2011) Associations and dissociations between default and self-reference networks in the human brain. Neuroimage 55, 225-232

70 Johnson, S.C. et al. (2002) Neural correlates of self-reflection. Brain $125,1808-1814$

71 Sajonz, B. et al. (2010) Delineating self-referential processing from episodic memory retrieval: common and dissociable networks. Neuroimage 50, 1606-1617

72 Levine, B. et al. (2002) Aging and autobiographical memory: dissociating episodic from semantic retrieval. Psychol. Aging 17, $677-689$

73 Caramazza, A. and Mahon, B.Z. (2003) The organization of conceptual knowledge: the evidence from category-specific semantic deficits. Trends Cogn. Sci. 7, 354-361

74 Mahon, B.Z. and Caramazza, A. (2011) What drives the organization of object knowledge in the brain? Trends Cogn. Sci. 15, 97-103

75 Kintsch, W. (1980) Semantic memory: a tutorial. In Attention and Performance VIII (Nickerson, R., ed.), pp. 595-620, Lawrence Erlbaum Associates

76 Jacoby, L.L. and Craik, F.I.M. (1979) Effects of elaboration of processing at encoding and retrieval: trace distinctiveness and recovery of initial context. In Levels of Processing in Human Memory (Cermak, L.S. and Craik, F.I.M., eds), pp. 1-21, Lawrence Erlbaum Associates

77 Kopelman, M.D. and Kapur, N. (2001) The loss of episodic memories in retrograde amnesia: single-case and group studies. Philos. Trans. R. Soc. Lond. B: Biol. Sci. 356, 1409-1421

78 Cabeza, R. and St Jacques, P. (2007) Functional neuroimaging of autobiographical memory. Trends Cogn. Sci. 11, 219-227

79 Greenberg, D.L. and Verfaellie, M. (2010) Interdependence of episodic and semantic memory: evidence from neuropsychology. J. Int. Neuropsychol. Soc. 16, 748-753

80 Barsalou, L.W. (1988) The content and organization of autobiographical memories. In Remembering Reconsidered: Ecological and Traditional Approaches to the Study of Memory (Neisser, U. and Winograd, E., eds), pp. 193-243, Cambridge University Press

81 Funnell, E. (2001) Evidence for scripts in semantic dementia: Implications for theories of semantic memory. Cogn. Neuropsychol. 18, 323-341

82 Rosch, E. and Mervis, C.B. (1975) Family resemblances - studies in internal structure of categories. Cogn. Psychol. 7, 573-605

83 Medin, D.L. and Schaffer, M.M. (1978) Context theory of classification learning. Psychol. Rev. 85, 207-238

84 Nelson, K. (1983) The derivation of concepts and categories from event representations. In New Trends in Conceptual Representation:
Challenges to Piaget's Theory? (Scholnick, E.K., ed.), pp. 129-149, Erlbaum

85 Conway, M.A. (1990) Associations between autobiographical memories and concepts. J. Exp. Psychol. Learn. Mem. Cogn. 16, 799-812

86 Ryan, L. et al. (2008) Hippocampal activation during episodic and semantic memory retrieval: comparing category production and category cued recall. Neuropsychologia 46, 2109-2121

87 Sheldon, S. and Moscovitch, M. (2012) The nature and time-course of medial temporal lobe contributions to semantic retrieval: an fMRI study on verbal fluency. Hippocampus 22, 1451-1466

88 Svoboda, E. et al. (2006) The functional neuroanatomy of autobiographical memory: a meta-analysis. Neuropsychologia 44, 2189-2208

89 Rubin, D.C. (2006) The basic-systems model of episodic memory. Perspect. Psychol. Sci. 1, 277-311

90 Moscovitch, M. (1992) Memory and working-with-memory - a component process model based on modules and central systems. J. Cogn. Neurosci. 4, 257-267

91 Tulving, E. (1984) Précis of Elements of Episodic Memory. Behav. Brain Sci. 7, 223-238

92 Tulving, E. (1985) Memory and Consciousness. Can. Psychol. 26, 1-12

93 Gilboa, A. et al. (2006) Hippocampal contributions to recollection in retrograde and anterograde amnesia. Hippocampus 16, 966-980

94 Dalgleish, T. et al. (2007) Reduced specificity of autobiographical memory and depression: the role of executive control. J. Exp. Psychol. Gen. 136, 23-42

95 Maki, R.H. (1993) Knowledge of the Self: Is it special? In Advances in Social Cognition (Vol. 5) Srull, T.K. and Wyer, R.S.,eds In pp. 101110, Lawrence Erlbaum Associates

96 Jefferies, E. et al. (2011) Premorbid expertise produces categoryspecific impairment in a domain-general semantic disorder. Neuropsychologia 49, 3213-3223

97 Funnell, E. and Davies, P.D. (1996) JBR: a reassessment of concept familiarity and a category-specific disorder for living things. Neurocase 2, 461-474

98 D'Argembeau, A. and Mathy, A. (2011) Tracking the construction of episodic future thoughts. J. Exp. Psychol. Gen. 140, 258-271

99 D'Argembeau, A. and Demblon, J. (2012) On the representational systems underlying prospection: evidence from the event-cueing paradigm. Cognition 125, 160-167

100 McCloskey, M.E. and Glucksberg, S. (1978) Natural categories: well defined or fuzzy sets? Mem. Cogn. 6, 462-472

101 Hampton, J.A. (1979) Polymorphous concepts in semantic memory. J. Verb. Learn. Verb. Behav. 18, 441-461

102 Johnson, K.E. and Mervis, C.B. (1997) Effects of varying levels of expertise on the basic level of categorization. J. Exp. Psychol. Gen. 126, 248-277

103 Spitzer, M. et al. (1998) Functional magnetic resonance imaging of category-specific cortical activation: evidence for semantic maps. Cogn. Brain Res. 6, 309-319

104 Chao, L.L. et al. (1999) Attribute-based neural substrates in temporal cortex for perceiving and knowing about objects. Nat. Neurosci. 2, 913-919 